Pacific Journal of Mathematics

WO THEOREMS ON METRIC SPACES 


\title{
TWO THEOREMS ON METRIC SPACES
}

\author{
HSIEN-CHUNG WANG
}

1. Introduction. Let $E$ be a metric space with distance function $d$. The space $E$ is called two-point homogeneous if given any four points $a, a^{\prime}, b, b^{\prime}$ with $d\left(a, a^{\prime}\right)=d\left(b, b^{\prime}\right)$, there exists an isometry of $E$ carrying $a, a^{\prime}$ to $b, b^{\prime}$, respectively. In a recent paper [7], the author has determined all the compact and connected two-point homogeneous spaces. It is the aim of the present note to discuss the noncompact case, and prove a conjecture of Busemann which can be regarded also as a sharpening of a theorem of Birkhoff [1]. The results concerning the noncompact two-point homogeneous spaces are not as satisfactory as the results for the compact case; we have to assume certain conditions on the metric.

By a segment in a metric space $E$, we shall mean an isometric image of a closed interval with the usual metric. A metric space will be said to have the property $(L)$ if given a point $p$, there exists a neighborhood $W$ of $p$ so that each point $x(\neq p)$ of $W$ can be joined to $p$ by at most one segment in $E$. The following theorems will be proved:

THEOREM 1. Let $E$ be a finite-dimensional, finitely compact, convex metric space with property $(L)$. If $E$ is two-point homogeneous, then $E$ is homeomorphic with a manifold.

THEOREM 2. Let $E$ be a metric space with all the properties mentioned in Theorem 1. If, moreover, $\operatorname{dim} E$ is odd, then $E$ is congruent either to the euclidean space, the hyperbolic space, the elliptic space, or the spherical space.

Our Theorem 2 justifies the conjecture of Busemann [2, p. 233] that a twopoint homogeneous three dimensional S. L. space $[2, \mathrm{p} .78]$ is either elliptic, hyperbolic, or euclidean. It is to be noted that Theorem 2 no longer holds if $\operatorname{dim} E$ is even and greater than two. The complex elliptic spaces [7] and the hyperbolic Hermitian spaces ${ }^{1}[2$, p. 192] serve as counter examples.

Received May 25, 1951.

1 These spaces were first introduced by H. Poincaré, and then discussed by G.Fubini and E. Study. Following E. Cartan, we call these spaces the hyperbolic Hermitian spaces.

Pacific J. Math. 1(1951),473-480. 
2. Preliminary results. Throughout this note, by a Busemann space $[2, \mathrm{p} .11]$, we shall mean a finitely compact, convex metric space such that at each point $p$, there exists a neighborhood $W$ with the following property: given any two points $x, y$ of $W$ and any $\epsilon>0$, we can find a positive number $\delta<\epsilon$ for which a unique point $z$ exists so that

$$
d(x, y)+d(y, z)=d(x, z), \quad d(y, z)=\delta .
$$

It can easily be verified that the class of all two-point homogeneous, finitely compact, convex metric space with the property $(L)$ coincides with the class of all two-point homogeneous Busemann spaces. In the statements of our Theorems, we use the property $(L)$ instead of Busemann's axioms merely because it is, geometrically, easier to visualize.

Let $E$ be a Busemann space. We shall first see that each $d$-sphere ${ }^{1}$ of sufficiently small radius is locally connected. In fact, let $p$ be a point of $E$. We choose $\epsilon>0$ so small that each point $x$ with $0<d(p, x) \leq \epsilon$ can be joined to $p$ by one and only one segment. Let $K(p, \epsilon)$ be the $d$-sphere with center $p$ and radius $\epsilon$, and $R$ the totality of points $y$ with $0<d(p, y)<\epsilon$. Then evidently $R$ is an open set of $E$. Since $E$ is convex, $E$ must be locally connected. It follows then that $R$ is locally connected.

For each point $y$ of $K(p, \epsilon)$, we denote by $P_{y}(s)(0 \leq s \leq \epsilon)$ the is ometric representation of the segment joining $p$ to $y$. Let $J$ be the open interval $0<s<\epsilon$. By our choice of $\epsilon$, the mapping $h: K(p, \epsilon) \times J \rightarrow R$ defined by $h(y, s)=P_{y}(s)$ is a one-to-one mapping of the topological product $K(p, \epsilon) \times J$ onto $R$. Moreover, from Busemann's results $\left[2, I_{.}, \S 3\right]$ concerning the convergence of geodesics, we see immediately that $h$ is bicontinuous. This tells us that $K(p, \epsilon) \times J$ and $R$ are homeomorphic. Since $R$ is locally connected, $K(p, \epsilon) \times J$, and hence $K(p, \epsilon)$, is locally connected.

3. Proof of Theorem 1. Let $E$ be a metric space with all the properties mentioned in Theorem 1. From the above discussions, we know that for any point $p$ of $E$, the $d$-sphere $K(p, \epsilon)$ with sufficiently small radius $\epsilon$ is locally connected. Let $\Gamma$ be the group of all isometries of $E$, and $\Gamma_{p}$ the totality of all those isometries which leave $p$ invariant. In $\Gamma$, we introduce the topology as defined by van Dantzig and van der Waerden [4] (in fact, this is exactly the g-topology of R. Arens).

1 By a $d$-sphere we mean the totality of points equidistant from a fixed point with respect to the metric $d$. This should be distinguished from the $(n-1)$-sphere which stands for the $(n-1)$-dimensional topological sphere. 
Then $\Gamma_{p}$ forms a compact topological group [4]. Evidently, $\Gamma_{p}$ is a transformation group of $K(p, \epsilon)$ in the sense of liontgomery and Zippin. From the two-point homogeneity, $\Gamma_{p}$ is transitive on $K(p, \epsilon)$. Taking account of the finite dimensionality and local connectedness of $K(p, \epsilon)$ and the compactness of $\Gamma_{p}$, we can conclude [5] that $\Gamma_{p}$ is a Lie group, and hence $K(p, \epsilon)$ is locally euclidean (here as well as in what follows, locally euclidean is always used in the topological sense). The set $R$, being homeomorphic with the topological product of $K(p, \epsilon)$ and the open interval $J$, must be locally euclidean as well. Hence our space $E$ is locally euclidean at each point of $R$, and hence locally euclidean at all its points. Moreover, $E$ is obviously separable and connected. It follows then that $E$ is homeomorphic with a manifold.

4. The structure of $d$-spheres. Before proving Theorem 2, we find it convenient to establish some more properties of the $d$-spheres.

LEMMA. Let $E$ be a metric space satisfying all the conditions in Theorem 2. Then each $d$-sphere with sufficient small radius is homeomorphic with the $(n-1)$ dimensional topological sphere where $\operatorname{dim} E=n$.

Proof. If $\operatorname{dim} E$ is equal to one, this is trivial. Now we shall assume that $n>1$. Let $p$ be a point of $E$, and $\epsilon$ so small that each point $x$ with $0<d(p, x) \leq \epsilon$ can be joined to $p$ by one and only one segment. Set $K(p, \epsilon)$ to be the $d$-sphere with center $p$ and radius $\epsilon$, and

$$
U=\{x \mid d(p, x)<\epsilon\} .
$$

We shall show first that $U$ is contractible to a point. Given each point $y$ of $K(p, \epsilon)$, let us denote by $P_{y}(s)$ the isometric representation of the segment joining $p$ to $y$. Then the pair $(y, s)$, where $y \in K(p, \epsilon)$ and $0 \leq s<\epsilon$, can be regarded as polar coordinates of points in $U$. For any real number $t$ with $0 \leq t \leq 1$, we define

$$
\phi\left[t, P_{y}(s)\right]=P_{y}(t s)
$$

We see immediately that $\phi$ is a well-defined mapping of the product $I \times U$, and

$$
\phi\left[1, P_{y}(s)\right]=P_{y}(s), \quad \phi(t, p)=p, \quad \phi\left[0, P_{y}(s)\right]=p,
$$

where $I$ denotes the closed interval $\{t \mid 0 \leq t \leq 1\}$. The continuity of $\phi$ can easily be verified. Thus $\phi$ gives a contraction of $U$ into the point $p$, and thus the homotopy group $\pi_{i}(U)$ vanishes for each $i$.

Now let us consider the set $R=U-p$. Since $U$ is an $n$-dimensional open 
manifold and $n>1$, the set $R$ is connected and has the same homotopy group $\pi_{i}$ as $U$ for all dimensions $i$ less than $n-1$. Thus $\pi_{i}(R)=0, i=1,2, \cdots, n-2$. On the other hand, we have shown in $\$ 1$ that $R$ is homeomorphic with the topological product $K(p, \epsilon) \times J$, where $J$ denotes an open interval. It follows then that $K(p, \epsilon)$ is connected and

$$
\pi_{i}[K(p, \epsilon)]=0, \quad i=1,2, \cdots, n-2 .
$$

From the proof of Theorem 1, we know that $K(p, \epsilon)$ is a homogeneous space of a compact Lie group. Its connectedness and its simply-connectedness imply that it is an orientable manifold.

Since both $K(p, \epsilon)$ and $J$ are manifolds, we have

$$
\operatorname{dim} K(p, \epsilon)+\operatorname{dim} J=\operatorname{dim} R=\operatorname{dim} E=n,
$$

and hence $\operatorname{dim} K(p, \epsilon)=n-1$. It follows immediately from (1) that $K(p, \epsilon)$ is a simply-connected homology sphere of even dimension $n-1$. Therefore [6] $K(p, \epsilon)$ is a topological sphere. The lemma is proved.

5. Proof of Theorem 2. Suppose $E$ to be a metric space with all the properties mentioned in Theorem 2. If $E$ is compact, then our Theorem 2 follows as a direct consequence of [7, Theorem VI]. Thus we can assume from now on that $E$ is not compact. We shall first show that $E$ is an open S. L. space in the sense of Busemann [2, p. 78]. To show this, it suffices [3, p.173] to establish that each geodesic is congruent to a euclidean line; for this, it suffices to demonstrate that given any two distinct points $x, y$ and any $k>0$, there exists a point $z$ so that

$$
d(x, y)+d(y, z)=d(x, z), \quad d(y, z)=k .
$$

In fact, since $E$ is finitely compact and noncompact, $E$ cannot be bounded. There exists then a sequence of points $p_{0}, p_{1}, p_{2}, \cdots$ with $d\left(p_{0}, p_{i}\right)$ tending to infinity. Thus we can choose $i$ so large that $d\left(p_{0}, p_{i}\right) \geq d(x, y)+k$. Let $\tau$ be a segment joining $p_{0}$ to $p_{i}$. Evidently there exist three points $x^{\prime}, y^{\prime}, z^{\prime}$ in $\tau$ such that

$$
d\left(x^{\prime}, y^{\prime}\right)+d\left(y^{\prime}, z^{\prime}\right)=d\left(x^{\prime}, z^{\prime}\right), \quad d\left(x^{\prime}, y^{\prime}\right)=d(x, y), \quad d\left(y^{\prime}, z^{\prime}\right)=k .
$$

From the two-point homogeneity of $E$, there is an isometry $f$ of $E$ carrying $x^{\prime}, y^{\prime}$ to $x, y$ respectively. Then we can see immediately that the point $z=f\left(z^{\prime}\right)$ has all the required properties. Thus $E$ is an open $S$. L. space.

Let $K(p, \epsilon)$ be the $d$-sphere with center $p$ and radius $\epsilon$, and $\Gamma_{p}$ the group of all 
isometries of $E$ which leave the point $p$ invariant. From the above lemma, we know that $K(p, \epsilon)$ is an $(n-1)$-sphere and $\Gamma_{p}$ a compact and transitive transformation group of $K(p, \epsilon)$. Moreover, it can easily be seen that $\Gamma_{p}$ is effective on $K(p, \epsilon)$. In our further discussions, we shall rule out the trivial case where $\operatorname{dim} E=n$ $=1$. Thus $K(p, \epsilon)$ is connected, and the identity component $\Gamma_{p}^{0}$ of $\Gamma_{p}$ forms a connected, compact, transitive, and effective transformation group of $K(p, \epsilon)$. Since $n-1$ is even, it follows [6] that $\Gamma_{p}^{0}$ is either isomorphic with the rotation group $R_{n-1}$ or Cartan's exceptional group $G_{2}$. We shall discuss these two cases separately.

Case A. Suppose $\Gamma_{p}^{0}$ to be isomorphic with the group $R_{n-1}$ of all rotations of the $(n-1)$-sphere. Let us represent $K(p, \epsilon)$ by the unit sphere in a certain $n$ dimensional euclidean space, and consider $R_{n-1}$ not only as a topological group but also as a transformation group of $K(p, \epsilon)$ in the usual sense. It is well known that $\Gamma_{p}^{0}$ and $R_{n-1}$ have the same topological type, that is, there exists a homeomorphism $\phi$ of $K(p, \epsilon)$ onto itself so that

$$
R_{n-1}=\phi \Gamma_{p}^{0} \phi^{-1}=\left\{\phi f \phi^{-1} \mid f \in \Gamma_{p}^{0}\right\}
$$

Since $n$ is odd, given any point $q$ of $K(p, \epsilon)$, there exists a rotation of period two which leaves fixed only $q$ and its diametrically opposite point. It follows then that for each point $q$ of $K(p, \epsilon)$, we can find a transformation $f$ in $\Gamma_{p}^{0}$ such that (a) $f$ is of period two, (b) $f$ leaves $q$ fixed, and (c) $f$ has only two fixed points on $K(p, \epsilon)$. Now let $g$ be any geodesic through $p$ in $E$. It intersects $K(p, \epsilon)$ at two points, say $q$ and $q^{\prime}$. We consider the transformation $f$ in $\Gamma_{p}^{0}$ having the above three properties (a), (b), and (c). Since $f$ is an isometry leaving fixed $p$ and $q$, it leaves the geodesic $g$ pointwise invariant. Moreover, this isometry $f$ cannot have any other fixed point, for otherwise $f$ would have some other fixed points on $K(p, \epsilon)$ besides $q$ and $q^{\prime}$. Thus $f$ is a reflection of $E$ about $g$. Since $p$ is an arbitrary point and $g$ an arbitrary geodesic through $p$, there exists a reflection of $E$ about each geodesic. From Schur's Theorem [2, p.181], it follows that $E$ is either hyperbolic or euclidean.

Case $B$. Suppose $\Gamma_{p}^{0}$ to be isomorphic with the exceptional group $G_{2}$. To discuss this case, we have to digress into a few properties of Cayley numbers. Let $1, e_{i}(i=1,2, \cdots, 7)$ be the units of Cayley algebra. The multiplication rule is given by 


$$
\begin{gathered}
e_{i} e_{i}=-1, \quad e_{i} e_{j}=-e_{j} e_{i}, \quad e_{1} e_{2}=e_{3}, \quad e_{1} e_{4}=e_{5}, \quad e_{1} e_{5}=e_{7}, \\
e_{2} e_{5}=e_{7}, \quad e_{2} e_{4}=-e_{5}, \quad e_{3} e_{4}=e_{7}, \quad e_{3} e_{5}=e_{5},
\end{gathered}
$$

together with the equalities obtained by cyclic permutation of the indices, Let

$$
\Theta=\left\{\sum_{i=1}^{7} x_{i} e_{l} \mid x_{\imath}=\text { real number, } \sum_{i=1}^{7}\left(x_{i}\right)^{2}=1\right\}
$$

be the totality of all the Cayley numbers with vanishing real part and with norm equal to unity. Evidently, $\Theta$ forms a 6-sphere, and each automorphism of the Cayley algebra carries $\Theta$ into itself. We can regard therefore the group $H$ of all automorphisms of Cayley algebra as a transformation group of $\Theta$ (the topology over $H$ is defined in the usual manner). Now $H$ acts effectively and transitively on $\Theta$. Moreover, it is known that $H$ is isomorphic with the exceptional group $G_{2}$.

For each $x=\sum_{i=1}^{7} x_{i} e_{i}$ in $\Theta$, we shall denote the Cayley number $x_{1}-\sum_{i=2}^{7} x_{i} e_{i}$ by $x^{*}$, and call it the symmetric image of $x$ with respect to $e_{1}$. It is evident that

$$
\left(x^{*}\right)^{*}=x, \quad x^{*}\left\{\begin{array}{ll}
=x, & \text { if } x= \pm e_{1}, \\
\neq x, & \text { otherwise. }
\end{array} \quad x \in \Theta\right.
$$

Moreover, by a direct calculation, we can show that given any two Cayley numbers $y, z$ in $\Theta$, there exists an automorphis $f$ in $\|$ such that

$$
f\left(e_{1}\right)=e_{1}, \quad f(y)=y^{*}, \quad f(z)=z^{*} .
$$

It is to be noted that this $f$ depends on $y$ and $z$. There is no automorphism of Cayley algebra which carries each $x$ in $\Theta$ into its symmetric image $x^{*}$.

Now we can proceed to the proof of Theorem 2. Since $\Gamma_{p}^{0}$ is isomorphic with the exceptional group $G_{2}, K(p, \epsilon)$ must be six-dimensional [6]. It is known that each transitive transformation group of the 6-sphere which is isomorphic with the exceptional group $G_{2}$ has the same topological type as $H .{ }^{1}$ Thus we can identify $\Theta$ and $K(p, \epsilon)$ in such a manner that $\Gamma_{p}^{0}$ and $H$ coincide. Let $x$ be a point of $K(p, \epsilon)$. It determines a ray $p x$, that is, the totality of points $u$ of $E$ for which either $d(x, u)$ $+d(u, p)=d(x, p) \overrightarrow{\text { or }} d(u, x)+d(x, p)=d(u, p)[2, p .76]$. For each nonnegative number $s$, we denote by $P_{x}(s)$ the point $u$ on the ray $\underset{\rightarrow}{p}$ with the property that

1 This follows as a direct consequence of $[6$, Lemma 6$]$. 
$a^{\prime}(p, u)=s$. Since $E$ is an open S. L. space, each point of $E$ other than $p$ can be represented in a unique way as $P_{x}(s)$, where $x \in K(p, \epsilon)$ and $s>0$. Let $y, z$ be any two points of $K(p, \epsilon)$, and let $y^{*}, z^{*}$ be, respectively, their symmetric images with respect to $e_{1}$ [note that we have identified $\Theta$ with $\left.K(p, \epsilon)\right]$. Then there exists a transformation $f$ in $\Gamma_{p}^{0}$ such that $f\left(e_{1}\right)=e_{1}, f(y)=y^{*}, f(z)=z^{*}$. Since $f$ is an isometry of $E$ and leaves $p$ fixed, we have, for any $s, s^{\prime} \geq 0$, the relations

$$
f\left[P_{y}(s)\right]=P_{y^{*}}(s), \quad f\left[P_{z}\left(s^{\prime}\right)\right]=P_{z^{*}}\left(s^{\prime}\right) .
$$

This tells us that

$$
d\left[P_{y}(s), P_{z}\left(s^{\prime}\right)\right]=d\left[P_{y^{*}}(s), P_{z^{*}}\left(s^{\prime}\right)\right] \quad\left(s, s^{\prime} \geq 0\right) .
$$

Now let us consider the mapping $h: E \rightarrow E$ defined by $h\left[P_{x}(s)\right]=P_{x^{*}}(s)$, where $x \in K(p, \epsilon)$ and $s \geq 0$. Equality (2) tells us that this mapping $h$ is an isometry of $E$. Moreover, from (1) we can see that $h$ is of period two and that $h$ has only two fixed points $e_{1}$ and $-e_{1}$ on $K(p, \epsilon)$. It follows then that $h$ is a reflection of $E$ about the geodesic joining $p$ and $e_{1}$. However, our space $E$ is two-point homogeneous so that there exists a reflection about every geodesic of $E$. From Schur's Theorem, we can conclude that $E$ is either hyperbolic or euclidean. Theorem 2 is hereby proved.

6. Remarks. In all the arguments, we use only the weaker two-point homogeneity; that is, there exists a number $\delta>0$ such that, for any four points $x, x^{\prime}$, $y, y^{\prime}$ with $d\left(x, x^{\prime}\right)=d\left(y, y^{\prime}\right)<\delta$. there exists an isometry of $E$ carrying $x, x^{\prime}$ to $y, y^{\prime}$ respectively.

The author wishes to express his thanks to Professor H. Busemann for his helpful suggestions concerning the proof of Theorem 2.

\section{REFERENCES}

1. G. Birkhoff, Metric foundations of geometry I, Trans. Amer. Math. Soc. 55 (1944), 465- 492 .

2. H. Busemann, Metric methods in Finsler spaces and in the foundation of geometry, Princeton, 1942.

3. - On spaces in which two points determine a geodesic, Trans. Amer. Math. Soc. 54 (1943), 171-184.

4. D. van Dantzig und B. van der Waerden, Ueber metrisch homogene Räume, Abh. Math. Sem. Hamburg 6 (1928), 291-296. 
5. D. Montgomery and L. Zippin, Topological transformation groups I, Ann. of Math. 41 (1940), 778-791.

6. H. C. Wang, A new characterisation of spheres of even dimension, Nederl. Akad. Wetensch. Proc. 52 (1949), 838-845.

7. Two-point homogeneous spaces, to appear in Ann. of Math.

Louisiana State University 


\title{
PACIFIC JOURNAL OF MATHEMATICS
}

\section{EDITORS}

\author{
HERBERT BUSEMANN \\ University of Southern California \\ R. M. RoBINSON \\ Los Angeles 7, California \\ University of California \\ Berkeley 4, California \\ E. F. BECKENBACH, Managing Editor \\ University of California \\ Los Angeles 24, California
}

\section{ASSOCIATE EDITORS}

R. P. DILWORTH
HERBERT FEDERER
MARSHALL HALL

\author{
P. R. HALMOS \\ HEINZ HOPF \\ R. D. JAMES
}

\author{
B $\emptyset$ RGE JESSEN \\ PAUL LÉVY \\ GEORGE PÓLYA
}

\author{
J. J. STOKER \\ E. G. STRAUS \\ KÔSAKU YOSIDA
}

\section{SPONSORS}

UNIVERSITY OF BRITISH COLUMBIA

CALIFORNIA INSTITUTE OF TECHNOLOGY

UNIVERSITY OF CALIFORNIA, BERKELEY

UNIVERSITY OF CALIFORNIA, DAVIS

UNIVERSITY OF CALIFORNIA, LOS ANGELES

UNIVERSITY OF CALIFORNIA, SANTA BARBARA

OREGON STATE COLLEGE

UNIVERSITY OF OREGON

\author{
UNIVERSITY OF SOUTHERN CALIFORNIA \\ STANFORD UNIVERSITY \\ WASHINGTON STATE COLLEGE \\ UNIVERSITY OF WASHINGTON

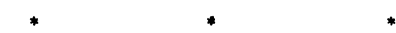 \\ AMERICAN MATHEMATICAL SOCIETY \\ NATIONAL BUREAU OF STANDARDS, \\ INSTITUTE FOR NUMERICAL ANALYSIS
}

Mathematical papers intended for publication in the Pacific Journal of Mathematics should be typewritten (double spaced), and the author should keep a complete copy. Manuscripts may be sent to any of the editors. All other communications to the editors should be addressed to the managing editor, E. F. Beckenbach, at the address given above.

Authors are entitled to receive 100 free reprints of their published papers and may obtain additional copies at cost.

The Pacific Journal of Mathematics is published quarterly, in March, June, September, and December. The price per volume (4 numbers) is $\$ 8.00$; single issues, $\$ 2.50$. Spécial price to individual faculty members of supporting institutions and to members of the American Mathematical Society: $\$ 4.00$ per volume; single issues, $\$ 1.25$.

Subscriptions, orders for back numbers, and changes of address should be sent to the publishers, University of California Press, Berkeley 4, California.

UNIVERSTTY OF CALIFORNIA PRESS • BERKELEY AND LOS ANGELES 


\section{Pacific Journal of Mathematics}

\section{Vol. 1, No. $3 \quad$ BadMonth, 1951}

R. P. Boas, Completeness of sets of translated cosines ............. 321

J. L. Brenner, Matrices of quaternions . ..................... 329

Edmond Darrell Cashwell, The asymptotic solutions of an ordinary differential equation in which the coefficient of the parameter is

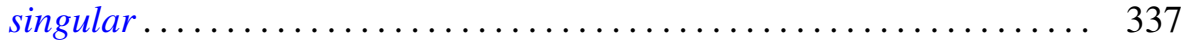

James Dugundji, An extension of Tietze's theorem ................ 353

John G. Herriot, The polarization of a lens ...................... 369

J. D. Hill, The Borel property of summability methods ............... 399

G. G. Lorentz, On the theory of spaces $\Lambda \ldots \ldots \ldots \ldots \ldots \ldots \ldots \ldots \ldots . \ldots \ldots$

J. H. Roberts and W. R. Mann, On a certain nonlinear integral equation of the Volterra type ................................. 431

W. R. Utz, A note on unrestricted regular transformations . . .......... 447

Stanley Simon Walters, Remarks on the space $H^{p} \ldots \ldots \ldots \ldots \ldots \ldots . . \ldots 55$

Hsien Chung Wang, Two theorems on metric spaces ............... 473 